sich auf der kalten Oberllächenspite : eine Wasserhaut bildet, auf die das gesamte Warmegefalle von dem Dampl anr Wandtemperatar entfullt. Dio Wasserbat baltet an der elnen-Seite an der Wand, die übrigen Scbiohten fllebèn nuter der Wlrkung der. Sobwere nach abwarte. Da die Siarke der Wasserhant jedentalls sebr gerlng ist - nach der Naseltschen Rechnung Bruchteile eines Millimeters - so haben wir es offenbar mit elner laminaren Sü̈wung zn tun. Eg tst nan plebt elnzusehen, warnm die.W.-Ua.-Zabl von Wasser an die metallische Wand ant der elaen Seite der Wand (wo nooh daza der Strömungezustand laminar isi) $7 \mathrm{mal}$ so grob, soln soll als an der anderen Beite (bei turbulenter Strömung).

Die Versuchsergebnisso von.Jogse müssen sich danach anders denten lassen; so daB die W.-D.-Zahl $k$ durch zwei annahernd gleich groBe Torme $\frac{1}{a_{1}}$ und $\frac{1}{\alpha_{l}}$, wobol $\alpha_{1}$ and $\alpha_{2}$ mittlere Werte haben, dargestellt wird. Es ersoheint sehr wahrscheinlioh, $\alpha_{a} B$ demnach die W. Ue.Zahl an Wasser höhere Werte hat, als vieltach blsher angenommex wurde. Eine umfassende experimentelle Untersuchung des Warmeïberganges an Flïssigkeiten erscheint dringend geboten, um dio Riuhtigkeit der theoretisohen Rechnungen prïfen zu könnon. Dle Schwierigkeiten, die sich bel der Verwendang, Yon Wasser durch Rostbildung und Kesselsteinansatz zeigen, legen es nabo, hierfur andero Flisaigkeiten, z. B. Oele, heranzuziebon.

\title{
Grundwasserströmung in einem abfallenden Gelände mit Abfanggraben,
}

\author{
Von L. HOPF und E. TREFFTZ in Aachen.
}

$\mathrm{D}$ ie Probleme der Grundwasserntrömong, bei denen die Gesobwindigkeit (Durohflußsenge pro Einheit des Querscbnittes in der Srkunde) proportional den aul dis Flilksigkeit wirkenden $\mathrm{K}_{\mathrm{r}} \mathrm{uat}_{\mathrm{i}}$ en gesetzt werden kann, fübren mathematisoh anf die gleichen Aufgaben (Integration der Gleichnng $\Delta u=0$ ) wie die Probleme der wirbellosen Strönung inkompressibler Fliissigkejten. Speziell die ebenen Problema sind daber den gleichen malhematischen Mrthoden der konformen Abblldung zogänglich, dio fitr die idealen Flüssigkeiten, z. B. bei der Strahlbildung, zur Lösung fübren. Io folgenden behandeln wir mit diesen Methoden eine Aulgabe, die unmittelbar der Praxis entnommen ist.

In den Mocisen" sijddeut-chlands stü̈ren beträchlliche Waskermengen mit ge. ringer Geschwindigkeit im Erdreich. Die freie Obertlache des Grundwassera lirgt der Eidoberfläche nahe, was eine Versumplung des Bodens zur Folge hat. Znm Zweoke der Trockenlegung solcher Moose und zugleich zur Ausnutzung der Wassermengen zur Kraftgewinnang sucht man dus Grundwasser in Graben abzufangen und wegzuleitrn. Relobt dieser Abfanggraben, icht bis zur Sohle der Grundwasser filhrenden Sobiobt, sondern tancht nur bis zu einer gew'sepn Tiefe ein, so erbebt sich die Frage, wellho Wasermengez ein solcher Graben der Guundwasserstı̈mung entzieht, welcbe Genchwinaigkeiten lokal suftreten, wie in einzelnen dis Stromtimen verlaufen, insbesondere die Oberfläche der Grundwasserströmung, und welohe konotruktiven Mabnahmen diese GröBen beoinflussen.

1. Physikalische und mathematische Grundlagen. Aul die Flïssigkeit wirken Druckgefälle, Schwere und Reibungskräfte Diese setzt wan proportional der Gesohwindigkeit nnd vernachliksigt alle Beschleunigungen. Derart erhält mán für die Gesohwindigkeitskomponenten den Ankatz:

oder vektoriell zusammengefabt:

$$
u=-k \frac{\partial p}{\partial x}, \quad v=-k\left(\frac{\partial p}{\partial y}+\gamma\right) .
$$

$$
\mathfrak{w}=-\operatorname{grad} \varphi \text {. }
$$

xobei das Potential $q=k(p+\gamma y)$ ist. Hierbei bedeuten:

$k$ eine vom Material der Wasser fübreuden Schicht abhängige Konstante (reziproke Roibungizahl),

$p$ den Druok,

$\gamma$ das fpezulische Gewiokt des Wassers,

$y$ die Höhe über einem Nullniveaa. 
Die Kontinultitggleichung ergibt dann filr das Potential $\varphi$ die Differentialgleichang $\Delta q=0$. In der gleichen Weise wis in der Theorie der idealen Flüesigkeiten oder therbaupt des ebenen Potentials fassen wir als den reellen Teil einer Fanktion $0=\varphi+i \psi$ der komplexen Variabeln $z=x+\hat{\imath} y$ anf, deren Imaginarteil $\psi$ (die Stromfanktion) auf den Stromlinien konstante Werte hat.

Die Grenebedingangen sind die folgenden:

Die undarcblässige Sohicht and die frele Oberfluche müssen Stromlinien -sein, d. h. es ist lăngs dlesen $\psi=$ konkt. An der freien Obenfläche muB auBerdem der Druok gleich dem konstanten Drucke der Atmosphäre soin, den wir gleich $\mathrm{Nall}$ setzen, d. h. es mub hier $q=k \gamma^{\prime} y=c y$ gelten. Schlieblich wird an der Grabensohle der Druck gleich dem hydrostatisohen Dracke (die' Bewegung des Wassers im Graben kann vernach- . lïssigt werden), also $\Phi=$ konst.

2. Der Abfanggraben. Dio Grundwasserströmung obne Abfanggraben verläbl, $\mathrm{Abb} 1$, geradlinig mit dem Gelallswinkel $\alpha$ der Soble. Die Grundwassersohle sei die Stromlinie $\psi=0$, der Grundwasserspiegel die Stromlinie $\psi=Q_{1}$, die Tiefo des

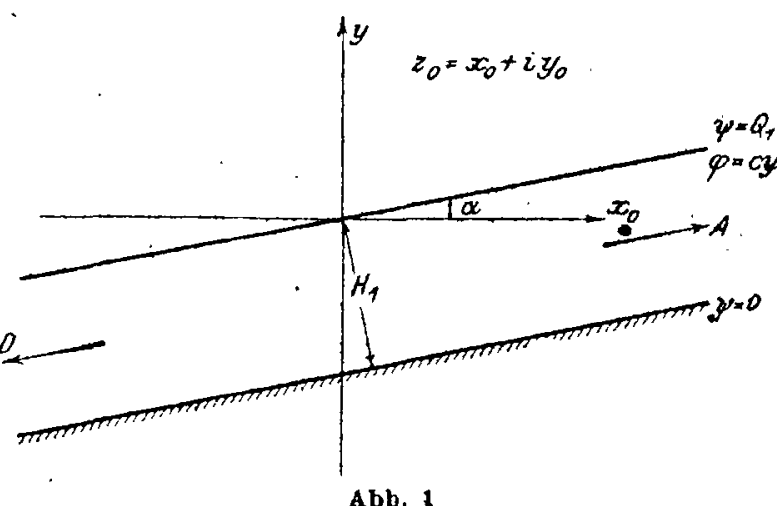

Abb. 1 Grandwassers sei $H_{1}$. Wir legen eine $x_{0}$ Achse horizontal, die $y_{0}$-Achse vertikal, den Nullpankt in den Spiegel. Der nnendlich ferne Punkt stromaufwärts sel $A$, stromabwarts $D$ genannt. Das Komplexe Potential wird

und

$$
\omega=z_{0} c \sin \alpha e^{-i \alpha}+i Q_{1} \quad . \quad . \quad . \quad . \quad . \quad . \quad . \quad(2)
$$

$$
\frac{Q_{1}}{\operatorname{css} \alpha}=H_{1}
$$

Nan sei die Grundwasferftrömung durch einen Abfanggraben gesiört, dessen Spiegel aut dem Niveau $y=0$ liege; der Punkt $x=0 \quad y=0$ liege auf dem Schnittpunkt des Grabenspiegels mit dem ungestörten Grondwasserspiegel, Abb. 2. Dis nndurchläsige Schicht, die die Grundwasserströmung nach unten begrenet, verlaufe geradlinig:

sie ist die Stromlinie $y=0$; $x \sin \alpha-y \cos \alpha=H_{1} \quad$. . . . . . . . . . (4), die freie Oberfladolie oberhalb des Grabens (stromaufwarts) $\psi^{\prime}=Q_{1}$, unterhalb des Grabens (stromabwärts) $\psi^{\prime}=Q_{2}$, wobei $Q_{2}<Q_{1} . \quad Q_{1}-Q_{2}$ ist die Wassermenge, welche im Graben fortgefuibrt und dem Grundwasserstrom entzogen wird. Längs der Grabensohle sei $q^{\prime}=0, \psi$ fälit von $Q_{1}$ aul einen Minimalwert $Q_{m}$ und wächet wieder bis $Q_{7}$. Die Schwierigkeit unseres Problems besteht genau wio bei

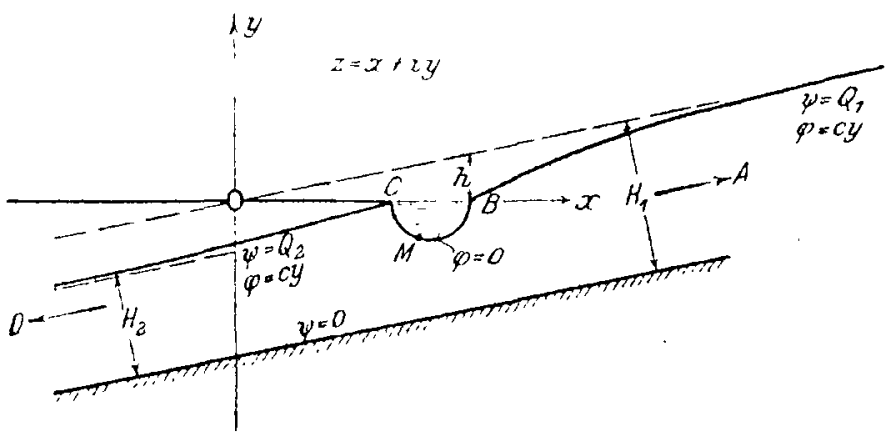

$A b b, 2$

den Strahla $f_{g a b e n}$ der klassischen Hydromechanik darin, daß wir die Oberilachen der Strömung nicht kennen. Genau wie dort fütren wir desbalb auch bier das komplexe Potential a als unabhängige, $z$ als abhängige Variable ein. In der a-Ebene, $\Delta b \mathrm{~b}$. 3 , ist $A B$ die Gerade $\psi=Q_{1}$ und $z w a r$ tür alle positiven Werte von $\varphi, C D$ die, Gerade $\psi=Q_{2}$ ftir alle negativen Werte von $q$ und dis Grundwassersohle $A D$ die $\varphi$.Achse. Dio Grabensohle $\varphi=0$ entsprioht dem Stüok $Q_{1}$ bis $Q_{m}$ und zurück bis $Q_{9}$ aut der $\psi$-Achse. Unser Gebiet in der a-Ebene besteht also aus einem Streifen, der links der $\psi$-Achse die Hohe

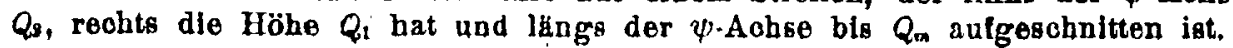




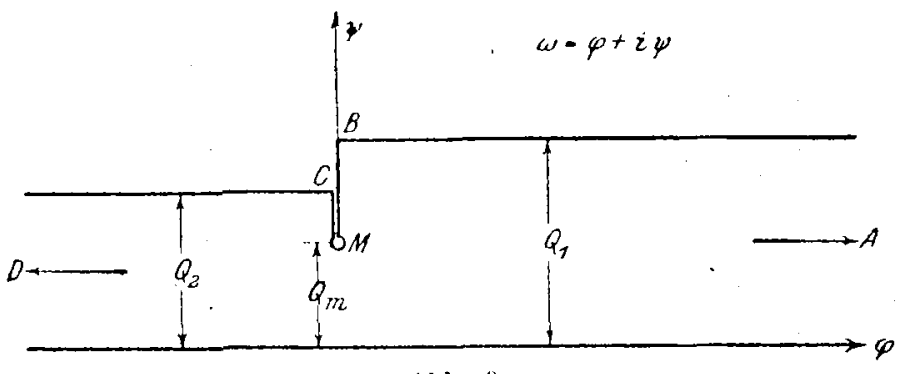

Abl. :

Für dieses festbegrenzte Gobiet haben wir nan $z$ als eine Funktion von $\omega$ so zu bestimmen, das länge der -Achse

$x \sin \alpha-y \cos \alpha=H_{1}$ (4) wird, rechts längs $\psi=Q_{1}$ únd links längs $\psi=Q_{3} \quad y=\frac{r}{c}$. und daß die Streifenbegrenzung auf der $\psi$-Achse in den Grabonumrib $y=F(x)$ über-

geht. Es liegt nahe, diese Aulgabe dadurch zu vereinfachen, das man

$$
z=z_{0}(\omega)+z_{1}(a) . . \quad . \quad . \quad .
$$

wobei

$$
z_{0}=\frac{\left.(\alpha)-i Q_{1}\right) e^{i a}}{c \sin a}
$$

die Strömung obne Abfanggraben darstellt, setzt und nun nur die Abweichung $z_{1}$ von der ungestörten Strömung zu bestimmen bleibt. Für $z_{1}$ ergeben sich die folgenden Randbedingangen, Abb. 4 .

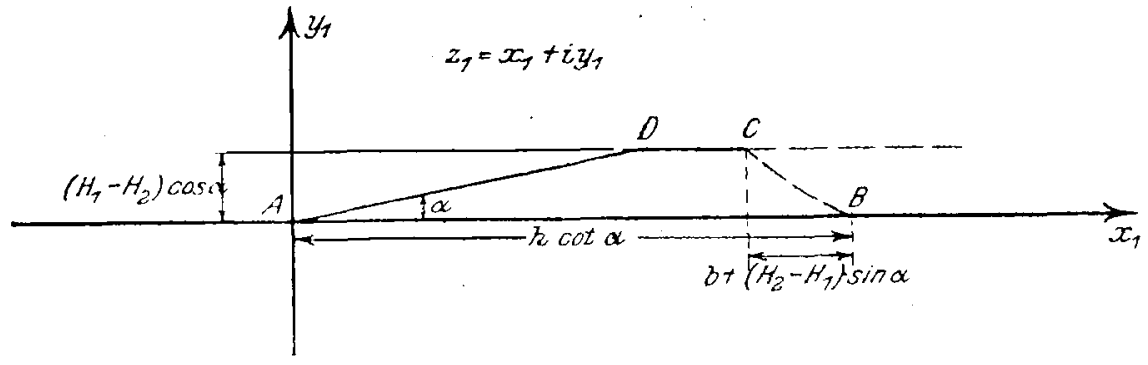

Abb. 4

Längs $A B$ ist $\psi^{\prime}=Q_{l}$, al6o nach (5):

$$
x+i y=\frac{q}{i \sin \alpha}(\cos \alpha+i \sin \alpha)+x_{1}+i y_{1} \text {. }
$$

Die Qrenzbedingung $y=\frac{\varphi}{i}$ verlangt mitbin:

$$
y_{3}=0 \text {. . }
$$

Im Punkt $A$ ist ofienbar auch $x_{1}=0$, denn in Unendlichen beeinjluBt der Graben die Strömung nicht. lä̈ngs $A D$ ist $\psi=0$ and $x \sin \alpha-y \cos \alpha=H_{1}$. Setzen wir hier $x$ und $y$ aus (5) mit $y=0$ ein, so wird:

$$
H_{1}=\frac{\sigma}{c} \cos \alpha+\frac{Q_{1}}{c} \sin \alpha-\frac{\varphi}{c} \cos \alpha+\frac{Q_{1}}{c} \frac{\cos ^{2} \alpha}{\sin \alpha}+x_{3} \sin \alpha-y_{1} \cos \alpha,
$$

daher, bei Benutzung von (3)

$$
x_{1} \sin \alpha-y_{1} \cos \alpha=0
$$

Längs $C D$ ist $\psi=Q_{2}$, also nach (5)

$$
y=\frac{\varphi}{c}+\frac{Q_{9}-\dot{Q}_{1}}{c} \operatorname{cotg} a+y_{1} \text {. }
$$

Da $y=\frac{\varphi}{c}$ sein soll, muB

$$
y_{1}=\frac{Q_{1}-Q_{2}}{c} \operatorname{cotg} \alpha
$$

sein.

3. Herstellung der Abbildung. Botrachten wir in der $z_{1}$-Ebene das Gebiet, in das aneer Streifen der $\infty$-Ebene übergefïhrt wird, so gehen die horizontalen Begrenzungen tunseres Streifens in die mit gleichen Buchstaben bezeichneten Strecken der $z_{i}$ Ebene tiber. Die Verbindung der Pankte $C$ and $B$, die dem Grabenumrib entepricht, 
bleibt auf der $z_{1}$-Ebene noch unbestimmt. Wir vereinfachen die Anfgabe dadurch, daB wir nicht den Grabenumriß vorgeben und dio zugehörige Strömung anfenchen, sondern die Größen $Q_{1}, Q_{2}$ und $Q_{m}$ geben und hierzu elne Grabenform aufsuchen. Zu diesem Zweck untersuchen wir zunächst den Zusammenhang zwisohen der Grabenbreite $b$, der Absenkung $h$ am Punkte $H$, der Absenkung im Unendlichen $H_{2}-B_{3}$ und den Abmessungen des abzubildenden Stroifens in der $z_{1}$-Ebene. Aus Abb. 2 folgt für die Absenkung $h=x(B) \operatorname{tg} a$, also, wenn wir in (5) fuir den Punkt $B \quad=0, \psi=Q_{1}$ einsetzen $\left(x_{0}=0\right)$,

$$
h=x_{1}(B) \operatorname{tg} \alpha . \quad . \quad . \quad . \quad . \quad . \quad . \quad . \quad . \quad . \quad . \quad(9),
$$

soda $B$ also die Strecke $A B$ in der $\tilde{\alpha}_{1}$-Ebene $x_{1}(B)=h \operatorname{cotg} \alpha$ wird $\left(9^{\prime}\right)$.

Der Unterschied der Abszissen $x_{1}(B)-x_{1}(C)$ rechnet sich aus (5), wenn wir für $B \varphi=0, \psi=Q_{1}$, für $C q=0, \eta=Q_{2}$ einsetzen und berücksichtigen, da $B$ die Grabenbreite $b=x(B)-x(C)$ ist:

dabei ist analog zu $H_{1}$

$$
x_{1}(B)-x_{1}(C)=b+\left(H_{1}-H_{2}\right) \sin \alpha . . . \quad \text {. . (10) }
$$

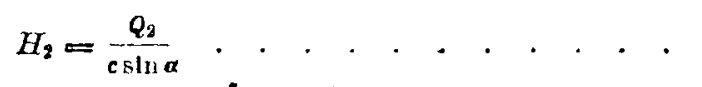

die Grundwassertiefe im Unendlichen stromabwäts."

Mit Benuizung dieser Abkürzung folgt ferner aus (6) und (8)

$$
y_{1}(D)=y_{1}(C) \doteq\left(H_{1}-H_{2}\right) \cos \alpha
$$

Das mathematisobe Problem, die w-Ebene auf die $z_{1}$-Ebene abzubilden, lösen wir durch Einführning einer Hilfsvariablen $\zeta$, Indem wir sowohl unseren Streifen in der $\omega$-Ebene als auch das Gebiet der $i_{1}$-Ebene anf den ersten Quadranten der $\zeta$-Ebene abbilden. Dabef soll der Punkt $B$ ins Unendliche rücken, $C$ in den Nullpunkt fallen, $A$ und $D$ auf der positiv reellen, $M$ auf der positiv imaginaren Ache liegen.

$$
(C: \zeta=0, B: \zeta=\infty, A: \zeta=a, D: \zeta=d, M: \zeta=i m) \text {. }
$$

Die Abbildung der a-Ebone aul den ersten Quadranten der $\zeta$-Ebene wird geliefert duroh die Formel

oder wenn

$$
\text { a) }==\frac{Q_{1}}{\pi} \ln \frac{a+\zeta}{a-\zeta}-\frac{Q_{1}}{\pi} \ln \frac{\zeta+d}{\zeta-d} . . . \quad . \quad . \quad . \quad . \quad .
$$

(Giehe Abb. 5) gesetzt wird,

$$
\lambda_{1}=\ln \frac{r_{1}^{\prime}}{r_{1}}, \lambda_{3}=\ln \frac{r_{2}^{\prime}}{r_{2}}
$$

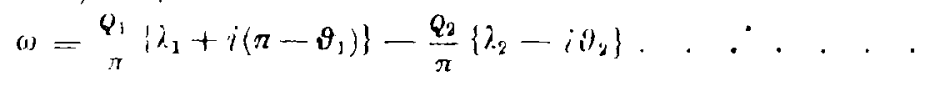

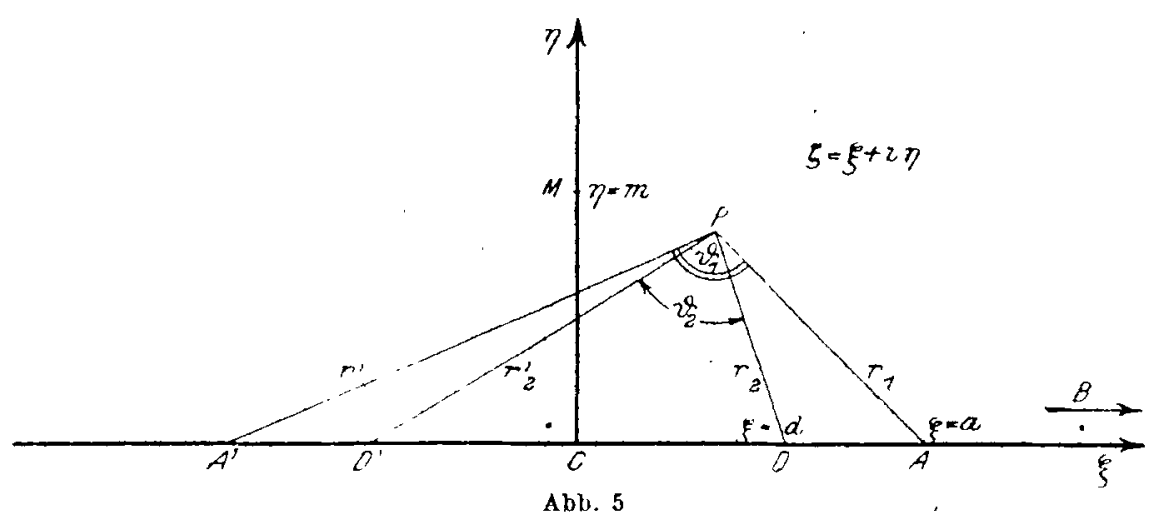

In der letzten Form können wir die Richtigkeit unserer Formel unmittelbar einsehen: Für alle positiv reollen $\zeta$ sind $\lambda_{1}$ und $\lambda_{3}$ beide positi $\nabla$, im Unendlichen $(B)$ und auf der ganzen positiven $\eta$-Achsp ist $\lambda_{1}=\lambda_{2}=0$. In $A$ wird $\lambda_{1}$, in $D \lambda_{2}$ unendlich. Auf der Strecke $A B$ ist $\vartheta_{1}=\vartheta_{2}=0$; auf $A D \vartheta_{1}=\pi, \vartheta_{2}=0 ;$ auf $C D \vartheta_{1}=\vartheta_{2}=\pi$, auf der positiven $\eta$ Achse gehen $\vartheta_{1}$ und $\vartheta_{3}$ von $\pi$ im Nallpunkt $(C)$ bis 0 im Unendlichen $(B)$. Es wird also 
Lenge $A B: \psi=Q_{1} \varphi$ durchlkuft alle posittren Werte von $0(B)$ bie $+\infty(A)$

- $A D: \psi=0 \quad q \quad$ "Werto von $+\infty(A)$ bis $-\infty(D)$,

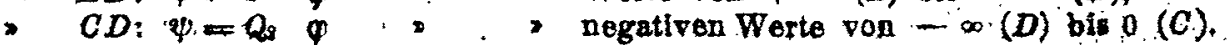

"der Imaginkien Achse wird $\varphi=0$.

Es wird also daroh (14) der erste Quadrant dor ל̧-Ebene in der Tat in den gegobenen Streifen der a-Ebene ibergeliuhrt.

4. Bestimmung der Konstanien. Um die relative Lage der Pankte $M$, $A$ und $D$ auf der $\zeta$-Ebeno zu bestimmen, berüoksiohtigen wir, das $\psi$ in $M$ ein Minimum hat; es mub also dort $\frac{\partial \psi}{\partial \eta}=0$ sein, d. $h$.

$$
-\frac{Q_{1}}{\pi} \frac{\partial \vartheta_{1}}{\partial \eta}+\frac{q_{2}}{\pi} \frac{\partial \vartheta_{1}}{\partial \eta}=\dot{0}
$$

Lange der $\eta$-Achse lot:

$$
\cot \frac{1}{2} \theta_{1}=\eta / a, \quad \cot \frac{1}{2} \eta_{2}=\eta / a .
$$

Wenn tür den Punkt $M$ wird, so ist dort

$$
\vartheta_{1}=\theta_{1 ;} \quad \quad \theta_{2}=\theta_{y_{m}}
$$

$$
\frac{\partial \vartheta_{1}}{\partial \eta}=-\frac{\sin \vartheta_{1 m}}{m}, \quad \frac{\partial \vartheta_{2}}{\partial \eta}=-\frac{\sin \vartheta_{g m}}{m}
$$

Daher wird:

$$
\frac{Q_{1}}{Q_{3}}=\frac{\operatorname{pin} \vartheta_{9 n}}{\sin \vartheta_{10}}
$$

Aus dieser Beziehung und

$$
Q_{m}=\frac{Q_{1}}{\pi}\left\{\pi-\theta_{1 m}\right\}+\frac{Q_{2}}{\pi} \theta_{2 m} \ldots \ldots, \ldots . . .
$$

folgt die relative Lage von $A$ und $D$ gegen $M$.

Damit haben wir den Zusammenhang von os and $\zeta$ gefunden. Um nun das Gobiet der $z_{1}$.Ebene ebenfalls auf dea ersten Quadranten der $\zeta$-Ebene abrubilden, setzen wir

oder

$$
\frac{d \varepsilon_{1}}{d \zeta}=\frac{r(\zeta)}{(\zeta-a)^{\alpha / \pi}(\zeta-a)^{1-a / \pi}} \cdot . . . . . . .
$$

$$
z_{1}=\int_{a}^{\vdots} \frac{f(\zeta) d \zeta}{(\zeta-a)^{a / \pi}(\zeta-a)^{1-\sigma / \pi}} .
$$

Darin ist $f(\zeta)$ eine Funktion, die fïr positiv reelle $\zeta=\xi$ positiv, im ganzen ersten Quadranten regultir und im Unendlichen Nall ist. Durch Verfigung über diese Funktion $f(\zeta)$ können wir die Grabenform variieren resp. unsere Löвung einer vorgeschriebenen Grabenform anpassen. Daß (17) and (18) wirklioh die Lösung lieferm, erkennen wir folgendermaßen: Bezeichnen wir mit $r_{1}$ and $r_{8}$ die Entfernungen von den Punkten $A$ und $D$, so ist

$$
\begin{aligned}
& \text { langs } C D: \quad \zeta-\alpha=r_{2} e^{i \pi}, \zeta-a=r_{1} e^{i \pi} \text {, also: } \frac{d \varepsilon_{1}}{d \zeta}=r_{2}^{-\alpha / \pi} e^{-i \alpha} r_{1}^{\alpha / \pi-1} e^{i(a-\pi)} f(\xi) \\
& \text { " DA: } \zeta-d=\tau_{2}, \zeta-a=r_{1} e^{i \pi} \text {, also: } \frac{d z_{l}}{d \zeta}=r_{9}^{-a / \pi} r_{1}^{\alpha / \pi-1} e^{i(a-\pi)} f(\xi) \\
& \text { DAB: } \zeta-d=r_{0}, \zeta-a=r_{1} \text {, also: } \frac{d z_{1}}{d \zeta}=r_{2}-a / \pi r_{1}^{a / \pi-1} f(\underline{\zeta}) \text {. }
\end{aligned}
$$

Da hier $d \zeta=d \xi$ reell ist, so folgt, wenn $d R$ das posillv reelle Dilferential $d R=r_{4}^{-\alpha / \pi} r_{1}^{(\alpha / \pi-1)} f(\xi) d \xi$ bedeutet:

$$
\begin{aligned}
& \text { längs } C D: \quad d z_{1}=-d \boldsymbol{R} \text {, } \\
& \rightarrow D A: d z_{1}=-e^{i a} d R \\
& \text { - } A B: \quad d z_{1}=+d R \text {. }
\end{aligned}
$$

Es werden also in der Tat die drei Abschnitte der 5 -Achse in der $z_{1}$-Ebene auf zwei horizontale Geradensttioke und eines von der Neigung $\alpha$ abgebildet, wie es verlangt war. 
5. Anpassung an die Grabenform. Daroh nnsere Abbildang geht die $\eta$-Aohse in die Grabenform iber. Für $f \zeta$ ) machen wir den Ansatz:

$$
f(\zeta)=\frac{A_{1}}{\zeta+e l}+\frac{A_{2}}{(\zeta+e l)^{2}}+\ldots
$$

wobel wir nos für die praktisohe Rechnung auf ewei Glieder besobrtnken werden. Die GröBe $l$ ist dabol willkürlich, da wir jede Funktion $f(v)$ im ersten Quadranten in der Form (19) annhhern können. Dle Koeflizienten $A_{1}$ und $A_{2}$ bestimmen sich ans den Grabenabmessungen.

Die Absenkung des Grabens ist gegeben durch (9):

$$
h=x_{1}(B) \operatorname{tg} \alpha=\operatorname{tg} \alpha \int_{a}^{\infty} \frac{\rho(\zeta) d \xi}{(\zeta-\alpha)^{\alpha / \pi}(\zeta-a)^{1-\alpha / \pi}} .
$$

Dieses Integral IaBt sich einfach auswerten, wenn man berücksichtigt, dab $\frac{a}{\pi}$ eine sehr kleine Größe (etwa $10^{-3}$ ) ist. Wir können also überall anßer in der un: mittelbaren Umgebung der Pankte $A$ and $" D$

$$
(\zeta-d)^{2 / \pi}=1 \text { und }(\zeta-a)^{1-a / \pi}=\zeta-i a
$$

setzen. Bel Auswertung des Integrals kommt es nur aut die unmittelbare Dmgebung des Panktes $A$ an. Man teilt das Integral in ein Integral von $a$ bis $a+\varepsilon$ und eines von $a+\varepsilon$ bis $\infty$ and behalt nur aus dem ersten einen Ausdruck von der Größenordnung $\frac{\pi}{a .}$ übrig. Es wird

$$
h=\pi f(a)+\text { GröBen von der Ordnung } \frac{\alpha}{\pi}
$$

Um die,Beziehung zwischen der Absenkang $h$ und der abgelangenen Wassermenge $Q_{1}-Q_{2}$ za erhalien, berüoksichtigen wir, daB nach (11)

$$
y_{1}(J)=\left(H_{1}-H_{2}\right) \cos \alpha=\frac{Q_{1}-Q_{2}}{c} \cot \alpha .
$$

Da

$$
z_{1}(D)=\int_{a}^{d} \frac{r(\zeta) d \zeta}{(\xi-d)^{\alpha / \pi}(\xi-a)^{1-\alpha / \pi}}=+e^{\alpha \alpha} \int_{d}^{a} \frac{f(\xi) d \xi}{(\xi-d)^{\alpha / \pi}(a-\xi)^{1-\alpha / \pi}},
$$

wird

$$
\frac{Q_{1}-Q_{2}}{c} \cot c=\sin \alpha \int_{d}^{a} \frac{f(\xi) d \xi}{(\xi-d)^{a / \pi}(a-\xi)^{i-a / \pi}} .
$$

Dies Integral zerlegen wir in drei Tejle, von $d$ bis $d+\varepsilon$, von $d+\varepsilon$ bis $a-\varepsilon$ und ron $a-\ell$ bis $a$. Nar rom letzten Teil bleibt ein Glied von der Gröbenordnang $\frac{\pi}{a}$ übrig, so daB in erster sehr guter Nuberung

also

$$
\int_{d}^{a} \frac{\rho(\xi) d \xi}{(\xi-d)^{0 / \pi}(a-\xi)^{1-\alpha / \pi}}=\frac{\pi}{\alpha} f(a)
$$

$$
\frac{Q_{1}-Q_{2}}{\cos \alpha}=\lg \alpha \frac{\pi}{\alpha} f(a)=\pi f(a)
$$

Der Vergleich der beiden Formeln (20) und (21) ergibt

$$
\frac{Q_{1}-Q_{2}}{c \sin \alpha}=H_{1}-H_{2}=h
$$

oder

$$
\frac{Q_{1}-Q_{2}}{Q_{1}}=\frac{h}{H_{1}}
$$

Bis auf Größen von der Ordnung $\frac{n}{\pi}$ verbălt sich also die abgefangene Grnod. wassermenge za der gesamten in der Sohicht fließenden Menge wie die Absenkung des 
Spiegels nnter den ungestörten Grundwasserspiegel zar Tiefe der Schicht. "Die abgefangene Menge wird also in erster Ordnung von keiner andern Abmessung des Grabens beeinfabt als von der Absenkang, insbesondere hangt sie, von der Breite oder der Wassertiefe im Abfanggraben nioht ab. Als Näherangsformeln sind bisher in der Praxis gelegentlich verwendet worden

$$
\frac{Q_{1}-Q_{2}}{Q_{1}}=\frac{t}{H_{1}-h}, \quad \frac{Q_{1}-Q_{2}}{Q_{1}}=\frac{h+t}{H_{1}}(t=\text { Wassertiefo im Ablanggraben }) \text {. }
$$

Beide Furmeli goben dio Verbälnisse nicht riohtig wieder. Von der Tiele des Wassers im Graben büngt die abgefangene Menge nioht ab; denn entsprechend der Tiefe wird das in den Graben eingedrangene Wasser wieder ans dem Graben abgefübrt. Von der Tiele und Breite des Wassers im Graben hängt natürlich $Q_{m}$ ab; die elnströmende Menge wird durch alle diese Gröben beeinflubt, aber die Aasströmung in derselben Weige, so daB der Grad des Ablangens nur duroh $h$ reguliert wird.

6. Numerisches Beispiel. Mit Hilfe unserer. Formeln wollen wir lolgendes Beiepiel durchrechnen. Wir stellen uns die Aufgabe, in eine Grundwasserströmung von der Neigung 0,003 einen Graben einzuziehen, der die Hälfe des Grundwassers abtängt. Nehmen wir an, daB 5 vH der Grundströmung in den Graben mit elndringt, ihn aber wieder verläBt, so wird

$$
Q_{2}=0,5 Q_{1} \quad Q_{n}=0,45 Q_{1} .
$$

Wir wăhlen als $I_{4}$ ïngeneinheit die Tiefe $H_{1}$ der ungestörten Grandwasserströmung ; dann ist $H_{9}=0,5, H_{m}=0,45$ und nach Formel (22) auch $h=0,5$.

$\mathrm{Da}$ in der $\zeta$ - Ebene nur Verhältnisgrößen auftreten, so könuen wir den Punkt $M$ in den Prinkt $\zeta=i$ legen.

Aus den Formeln

und

ergeben sioh dann

$$
\frac{H_{1}}{H_{2}}=\frac{\sin \vartheta_{2 m}}{\sin \vartheta_{1 m}}
$$

$$
\pi H_{m}=H_{1}\left(\pi-\theta_{1 m}\right)+H_{2} \theta_{2 n}
$$

$$
\begin{array}{rlrl}
\vartheta_{1 m} & =151^{\circ} & \vartheta_{2 m} & =104^{\circ} \\
a & =3,87 & d & =1,28 .
\end{array}
$$

Mit Hille dieser Zahlen ergeben aich (Abb. 5, Formel 14)

$$
\frac{q}{c \sin \alpha}=\frac{1}{\pi} \lambda_{3}-\frac{1}{2 \pi} \lambda_{2}, \quad \frac{\psi^{\prime}}{c \sin \alpha}=\frac{\pi-\eta_{1}}{\pi}-\frac{g_{2}}{2 \pi}
$$

Um das Strömungsbild in der $z$-Ebene zu finden, müssen wir zunächst $z_{0}=$ $\frac{\omega-i Q_{1}}{c \sin \alpha} e^{i \alpha}(2)$ reohnen, d. h.

$$
\begin{aligned}
& x_{0}=\frac{\varphi}{c \sin \alpha} \cos \alpha-\frac{y-Q_{1}}{c \sin \alpha} \sin \alpha-\frac{\varphi}{c \sin \alpha} \\
& y_{0}=\frac{\varphi}{c \sin \alpha} \sin \alpha+\frac{\psi-Q_{1}}{c \sin \alpha} \cos \alpha
\end{aligned}
$$

Das zweite Glied im Ausdruck fïr $x_{0}$ ist weggelassen, weil es kleiner als sin $\alpha$ ist, das erste Glied flir $y_{0}$ muß stehen bleiben, weil es für große $\uparrow$ von Bedeutung ist.

Zur Berechnung von $z=z_{0}+z_{1}$ (5) bleibt nooh das Integral:

$$
z_{1}-z_{1}(B)=\int_{\alpha}^{t} \frac{f(\zeta) a \xi}{(\zeta-d)^{\alpha / \pi}(\zeta-n)^{1-\alpha / \pi}} \ldots . . . . . .
$$

guszurerten.

Für $f(\zeta)$ machen wir den Ansatz

$$
f(\zeta)=\frac{A_{1}}{\zeta+l}+\frac{A_{2}}{(\zeta+l)^{9}}
$$

In dem Iategral (18) können wir, abgeseheo von der unmittelbaren Umgebang des Punktes $A(\zeta-d)^{a / \pi}=1(\zeta-a)^{1-a / \pi}=\zeta-a$ setzen und erhalten durch Partialbruchzerlegung:

$$
z_{1}-z_{1}(B)=\frac{\dot{A}_{3}}{(l+a)(\zeta+l)}+f(a) \ln \frac{\zeta-a}{\zeta+l}
$$


Non ist nach Formel (20) $f(a)=\frac{i}{\pi}=\frac{1}{2 \pi}$.

Willkürlich sind also zunächst noch $A_{2}$ und $l$. Damit eine positive Grabenbreite herauskommt, muß $l$ wesentlioh größer als $a$ sein. Wir haben $l=7,25$ gewählt und $A_{9}$ so bestimmt, daB $b=1 / 1$ wird, das ergibt

$$
\frac{A 2}{a+l}=0,242 \text {. }
$$

Es wird also

$$
\left.\begin{array}{l}
x_{1}-x_{1}(B)=\frac{1}{2 \pi} \ln \frac{r_{a}}{r_{l}}+0,242 \frac{\cos \vartheta_{l}}{\pi} \\
y_{1}-y_{1}(B)=\frac{1}{2 \pi} \Theta-0,242 \frac{\sin \vartheta_{l}}{r_{l}}
\end{array}\right\}
$$

wobei $n_{\text {I }}$ die Länge des Fabrstrahls vom Pankte $\zeta=-l$ nach $\zeta$ - bedeutet and $\vartheta_{l}$ der. Winkel dieses. Fahrstrabls gegen die ร-Achse ist. $\Theta$ ist der Winkel dieses Fahrstrahle gegen die Richtung von $\zeta$ nach $a$.

Daboi ist

$$
x_{1}(B)=h \cot \alpha=\frac{10^{8}}{6}, \quad y_{1}(B)=0 \text {. }
$$

In der Nähe von $\zeta=a, d, b$. in größerer Entfernung oberhalb des Grabens, gilt (24) nicht mehr.: Man mub von $A$ aus entwickeln und erhält:

$$
\begin{aligned}
z_{1}= & \frac{\pi}{a} f(a)(\zeta-a)^{a / \pi}=\frac{h}{a}(\zeta-a)^{a / \pi} \\
& =\frac{1000}{6}(\zeta-a)^{0,000956} .
\end{aligned}
$$

Die Resultate der rechnerischen Auswertung von ( 44$)$ and (25) siud in Abb. 6 and 7 wiedergegeben. Es bietet keine grundsătzlichen Sohwierigkeiten, die Parameter ron $f(\zeta)$ so za bestimmen, dab sich die Grabenform vorgegebenen Verbältnissen anpaßt. Wir, wollen aber nicht nảher ant diese Diskussion eingeben, da sle uns von geringem praktischen Interesse zu sein scheint. Die abgeführte Wassermenge und der Verlauf des Grundwasserspiegels, abgesehen von der unmittelbaren Umgebung des Grabens, sind von der Grabenform un-
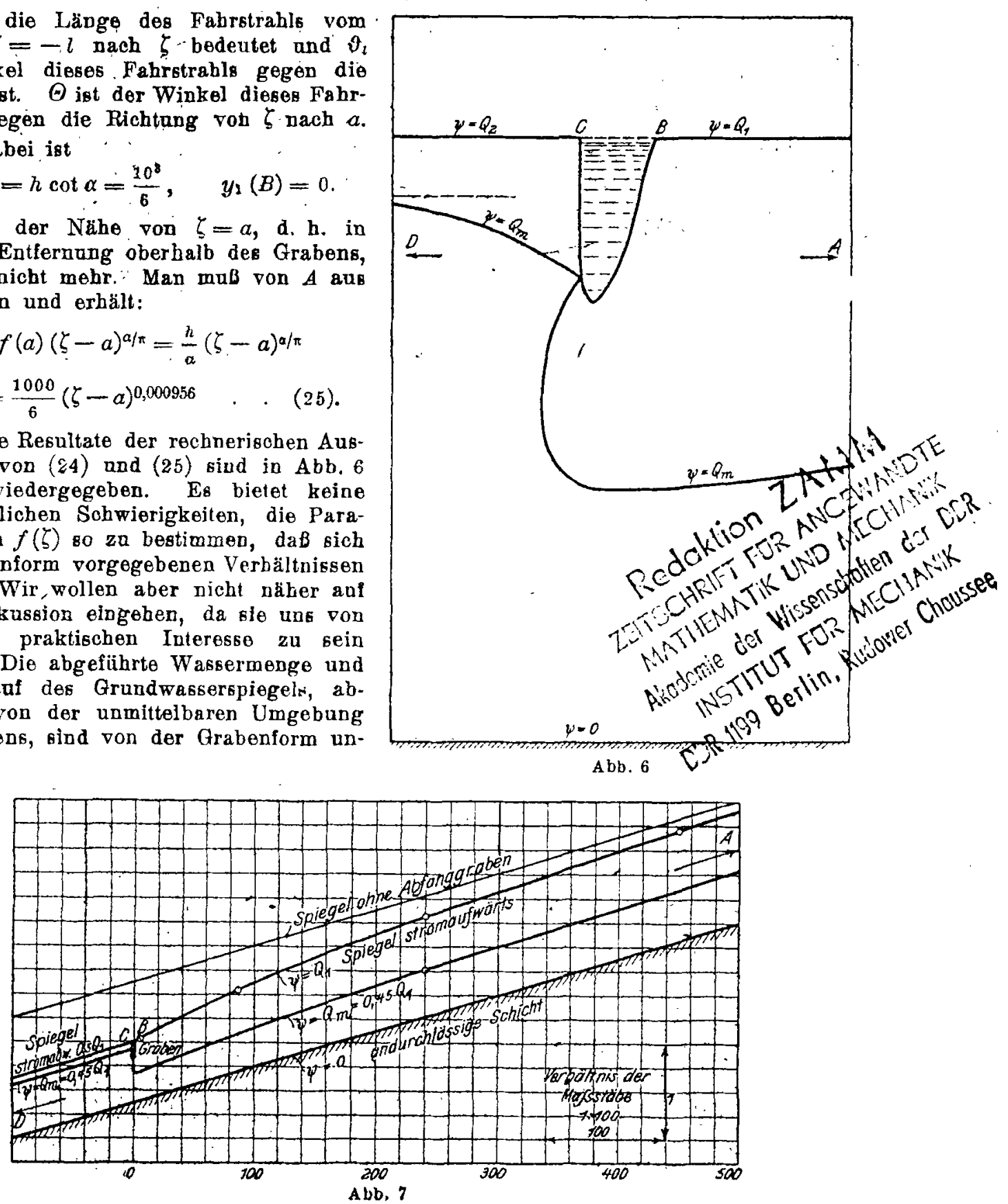
abbangig. Die Form des Grabens wird wesentlich duroh die Strömungsverbăltnisse Im Graben selbst bestimnt.

Znm Schlab ist noch folgendes zn bemerken: Unsere Rechnnng stellt insoferm einen Spezialfall dar, als wir den Grundwasserspiegel stromaufwäts am Graben in der Höhe des Grabenspiegels ansetzen. Es wäre an sich nicht ansgesohloseen, daß Wasser durch die Grabenwand oberhalb des Spiegels einträte und an der Grabenböschung herabslckerto. Dies wird dann eintreten, wenn man, ohne die abgeführte Menge $Q_{1}-Q_{z}$ zu ändern, duroh entsprechende Dimensionierung des Grabens den Splegel weiter senkt, als unsere Rechnung ergibt. Unsere Rechnung liefert also: die kleinste Wassermenge bei gegebener Absenkung oder umgekehrt die kleinste Absenkang bei gegebener Wassermenge. 87

\title{
Das Problem der Iterationen.
}

\author{
Von R. v. MISES in Berlin.
}

W er dem Ablauf einer vom Zufall beherrschten Reihe von Alternativ-Vorgängen (Kopf- oder Adlerspiel, Geschlechtsrerhältnis der Geburten usw.) seine Aufmerksamkeit zuwendet, wird unmittelbar auf die Beobachtung der längeren oder kïrzeren Wiederholungsfolgen desselben Ergebnisses hingewiesen. Alle sogenannten Spielsysteme knïpfen an diese Wiederholungen oder Iterationene an und auch in den Wahrscheinlichkeits-Betrachtungen des gewöhnlichen Lebens mißt man ihnen die größte Bedeutung bei. Wenu boim Spiel auf sgrad oder ungrad* fünfmal nacheinander »grad* erschienen ist, moint man, das nächste Mal könne es nicht mehr so bleiben; umgekebrt geht eine weit verbreitete Ansicht dahin, daB auf einen Eisenbahnunfall stets noch zwei weitere folgen (hier lautet die Alternative: glücklicher oder unglücklicher Ausgang einer Eisenbahnfahrt). Beide Anschauungen haben auch schon ihre wissenschaftliche Vertretung gefunden: Der Philosoph $\mathrm{Karl}$ Marbe sucht in einem umfangreichen Werke "Die (Gleichförmjgkeit in der Welt«') den Nachweis zu erbringen, daß lange Iterationen tatsächlich seltener vorkommen, als es die Wahrschoinlichkeitsrechnung erwarten läßt, und der Biologe Paul Kammerer häuft in seinem "Gesetz der Serie "\#) eine Menge von Einzelbeobachtungen, um zu zeigen, daB wenigstens kurze Wieder. holungsfolgen weit häufiger auftreten, als man durchschnitlich erwarten sollte.

In der vorliegenden Arbeit wird zunächst das mathematischo Problem der Iterationen, das bisher nicht orledigt war ${ }^{3}$ ), einer Lösung zugeführt, d. b. die allgemeine Frage folgender Art heantwortet: Welches ist die Wahrscheinlichkeit $w$ dafür, dab bei $n$ Würfon mit einer Münze gerade $\boldsymbol{x}$ Iterationen der Länge $m$, also $z$. B. bei tausend Wiirfen drei Folgen von genan sechs gleichen Ergebnissen eintreten? Die Antwort lautet hier, wenn die Wahrscheinlichkeiten der befden möglichen Einzelergebnisse, Kop! oder Adler, zu $1 / 2$ angenommen werden, $w=0,032$. Allgemein werden wir zeigen, das bei genügend grobem $n$ :

$$
w(x)=\frac{e^{-a} a^{x}}{x !}
$$

gilt, wenn $a$ die aus dem Beobachtungsumfang $n$, der Iterationslänge $m$ und den Grandwahrscheinlichkeiten zu ermittelnde Erwartungszahl von Iterationen bedentet. Von dieser konkreten Lösung aus ergibt sich erst die Möglichkeit, das anr Begründung jener populären Ansichten Gesagte einwandfrel zu bearteilen.

Wie bei allen derartigen Problemen der Wahrscheinlichkeitsrechnung bietet die Lösung, sobald einmal die Aufgabe klar formuliert (1) and auf die vier Grundaufgaben der Wahrsoheinlichkeitsrechnung zurückgeführt ist, keinerlei grundsutzliche Schwierig: keiten. Man kann ein Verfahren angeben (2), das die gesnchte Wahrscheinlichkeit in einer endlichen Anzahl von Sohritten aufzufinden gestattet. Wird aber der Umfang $n$ der Beobachtungsreihe sehr groß, so verliert dieses Verfahren seine praktische Anwendbarkeit und die Lösung golingt nur an einem Umweg: Man muB die sogenannten $*$ Momente der gesuchten Wahrscheinlichkeitsfunktion einfuihren (3), für die sich eine gerade bei großem $n$ sehr brauchbare Abschätzung finden läbt (4). Ans den Orenzwerten der Momente kann man dann auf Grund nenerer Ergebnisse über das Momentenproblem. auf die zu ermittelnde Fanktion selbst schließen (5 and 6). Der Vergleioh mit den von

1) 2 Bde. Mopchen 1916/1919. - 2) Stuttgart u. Berlin 1918. - T) Ueber die Literatar vergl. L. v. Bortkiewicz, Dfe Jeratlonen, Berlin 1917, sowie meinen Anfratz in Die Noturwissessoliaftena, $7,1919,8.168 \mathrm{HP}$. 